\title{
Research
}

\section{Effectiveness of early switch from intravenous to oral antibiotics in severe community acquired pneumonia: multicentre randomised trial}

\author{
Jan Jelrik Oosterheert, Marc J M Bonten, Margriet M E Schneider, Erik Buskens, \\ Jan-Willem J Lammers, Willem M N Hustinx, Mark H H Kramer, Jan M Prins, Peter H Th J Slee, \\ Karin Kaasjager, Andy I M Hoepelman
}

\begin{abstract}
Objectives To compare the effectiveness of an early switch to oral antibiotics with the standard 7 day course of intravenous antibiotics in severe community acquired pneumonia.

Design Multicentre randomised controlled trial.

Setting Five teaching hospitals and 2 university medical centres in the Netherlands.

Participants 302 patients in non-intensive care wards with severe community acquired pneumonia. 265 patients fulfilled the study requirements. Intervention Three days of treatment with intravenous antibiotics followed, when clinically stable, by oral antibiotics or by 7 days of intravenous antibiotics.

Main outcome measures Clinical cure and length of hospital stay.

Results 302 patients were randomised (mean age 69.5 (standard deviation 14.0), mean pneumonia severity score 112.7 (26.0)). 37 patients were excluded from analysis because of early dropout before day 3 , leaving 265 patients for intention to treat analysis. Mortality at day 28 was $4 \%$ in the intervention group and $6 \%$ in the control group (mean difference 2\%, 95\% confidence interval $-3 \%$ to $8 \%$ ). Clinical cure was $83 \%$ in the intervention group and $85 \%$ in the control group (2\%, $-7 \%$ to $10 \%$ ). Duration of intravenous treatment and length of hospital stay were reduced in the intervention group, with mean differences of 3.4 days (3.6 (1.5) v 7.0 (2.0) days; 2.8 to 3.9$)$ and 1.9 days (9.6 (5.0) v 11.5 (4.9) days; 0.6 to 3.2 ), respectively.

Conclusions Early switch from intravenous to oral antibiotics in patients with severe community acquired pneumonia is safe and decreases length of hospital stay by 2 days.
\end{abstract}

Trial registration Clinical Trials NCT00273676.

\section{Introduction}

When patients are first admitted to hospital with community acquired pneumonia, antibiotics are usually given intravenously to provide optimal concentrations in the tissues. Conventionally, intravenous treatment is continued until definite clinical cure. A switch to oral antibiotics may allow early discharge and reduce drug costs, but may increase the rate of treatment failure, readmission, and death; it may also increase the workload for family members or healthcare professionals outside the hospital.

The concept of early transition from intravenous to oral antibiotics in the treatment of community acquired pneumonia has been evaluated in mild to moderately severe disease and rarely in randomised trials. For patients with more severe forms of the disease, effects on outcome and length of hospital stay have not been determined in randomised trials. We conducted a multicentre randomised trial to evaluate the effectiveness of an early switch from intravenous to oral antibiotics compared with a seven day intravenous treatment regimen in patients with severe community acquired pneumonia.

\section{Patients and methods}

\section{Study design}

We performed a multicentre, randomised open label clinical trial in two university medical centres and five teaching hospitals in the Netherlands. Patients were randomised to the intervention group, where clinically stable patients ${ }^{1}$ were switched from intravenous to oral antibiotics on the third day in hospital to complete a total of 10 days of antibiotic treatment, or to the control group, where patients received a standard regimen of seven days of intravenous treatment. Additional antibiotic treatment thereafter was left to the discretion of the doctor. The attending consultant chose the antibiotics on the basis of Dutch treatment guidelines. ${ }^{2}$

Primary outcome measure was clinical cure. Secondary outcome measure was the length of hospital stay.

\section{Patients}

Patients $\geq 18$ years with severe community acquired pneumonia admitted to general hospital wards were eligible for inclusion. We defined pneumonia as a new or This is the abridged version of an article that was posted on 10.1136/bmj.38993.560984.BE
Editorial by Mills and Laing

Department of Internal Medicine and Infectious

Diseases, University Medical Centre, PO Box 85500,3508

GA Utrecht,

Netherlands

Jan Jelrik

Oosterheert

research associate

Marc J M Bonten

professor of infectious

diseases

Margriet M E

Schneider

infectiologist

Andy I M

Hoepelman

professor of medicine

Julius Centre for

Health Sciences

and Primary Care,

University Medical

Centre

Erik Buskens

associate professor of

epidemiology

Department of

Pulmonology,

University Medical

Centre

Jan-Willem J

Lammers

professor of

pulmonology

Department of

Internal Medicine,

Diakonessenhuis,

Utrecht

Willem M N

Hustinx

infectiologist

Department of

Internal Medicine,

Meander Medical

Centre, Amersfoort

Netherlands

Mark H H Kramer

internist/haematologist

continued over

BMJ 2006;333:1193-5 
Department of Division of Infectious Diseases, Tropical Medicine and Aids, Academi Medical Centre, Amsterdam, Netherlands Netherlands

Department of Internal Medicine,

St Antonius

Hospital,

Nieuwegein,

Netherlands

Peter H Th J Slee internist

Department of Internal Medicine, Rijnstate Hospital, Arnhem,

Netherlands

Karin Kaasjager internist

Correspondence to: A I M Hoepelman i.m.hoepelman@ umcutrecht.nl Internal Medicine,

progressive infiltrate on a chest radiograph plus at least two other criteria (cough, sputum production, rectal temperature $>38^{\circ} \mathrm{C}$ or $<36.1^{\circ} \mathrm{C}$, auscultatory findings consistent with pneumonia, leucocytosis $\left(>10^{9}\right.$ white blood cells/litre or $>15 \%$ bands), $\mathrm{C}$ reactive protein more than three times the upper limit of normal, or positive culture of blood or pleural fluid). Severe pneumonia was defined as pneumonia severity index class IV or V or fulfilling the American Thoracic Society criteria for severe community acquired pneumonia. ${ }^{4}$ See bmj. com for reasons for excluding patients.

Baseline, follow-up, and outcome measurements Patients were followed up for 28 days. On admission (day 0), we performed physical examination, chest radiography, and blood sampling for arterial blood gases, haematological analysis, and biochemical markers. We recorded demographic and clinical data and initial intravenous treatment. We evaluated clinical stability after three days of intravenous treatment in both groups and evaluated preset discharge criteria (temperature $<37.8^{\circ} \mathrm{C}$, oxygen saturation $>92 \%$, normal blood pressure, heart rate $<100 / \mathrm{min}$, respiratory rate $<25 / \mathrm{min}$, absence of mental confusion, and ability to take oral drugs) daily thereafter. Patients discharged within 28 days were asked to return 28 days after inclusion, for history, physical examination, blood chemistry analysis, and a chest radiograph.

We used questionnaires to measure the effect of early discharge on adverse events, compliance, and how the route of administration affected freedom of movement.

Treatment failure was defined as death, still in hospital at day 28 of the study, or clinical deterioration (increase in temperature after initial improvement or the need for mechanical ventilation, switch back to intravenous antibiotics, or readmission for pulmonary reinfection after discharge). Clinical cure was defined as discharged in good health without signs and symptoms of pneumonia and no treatment failure during follow-up."

\section{Microbiological analyses}

We used standard procedures to collect, culture, and evaluate sputum and blood samples. See bmj.com for details.

\section{Sample size and statistical analysis}

We initially set the sample size at 250 patients in each group on the basis of an expected cure rate of $85 \%$ in

Clinical outcome in multicentre randomised trial of early switch from intravenous to oral antibiotics in severe community acquired pneumonia. Intention to treat analysis. Values are number of patients (percentage) unless stated otherwise

\begin{tabular}{lccc} 
& \multicolumn{2}{c}{ Treatment group } & \\
\cline { 2 - 3 } Clinical outcome & $\begin{array}{c}\text { Intervention } \\
(\mathbf{n = 1 3 2})\end{array}$ & $\begin{array}{c}\text { Control } \\
\mathbf{( n = 1 3 3 )}\end{array}$ & Mean difference (95\% CI) \\
\hline Death after day 3 & $5(4)$ & $8(6)$ & $2 \%(-3 \%$ to $8 \%)$ \\
\hline Clinical cure & $110(83)$ & $113(85)$ & $2 \%(-7 \%$ to $10 \%)$ \\
\hline Clinical failure: & $22(17)$ & $20(15)$ & $-2 \%(-10 \%$ to $7 \%)$ \\
\hline Clinical cure but still in hospital & $9(7)$ & $6(5)$ & $-2 \%(-4 \%$ to $8 \%)$ \\
\hline Clinical deterioration & $8(6)$ & $6(5)$ & $-1 \%(-4 \%$ to $7 \%)$ \\
\hline Death & $5(4)$ & $8(6)$ & $2 \%(-3 \%$ to $8 \%)$ \\
\hline Clinical deterioration and death & $13(10)$ & $14(11)$ & $1 \%(-1 \%$ to $8 \%)$ \\
\hline $\begin{array}{l}\text { Mean (SD) length of hospital stay } \\
\text { (days) }\end{array}$ & $9.6(5.0)$ & $11.5(4.9)$ & $1.9(0.6$ to 3.2$)$ \\
\hline $\begin{array}{l}\text { Mean (SD) duration of intravenous } \\
\text { treatment (days) }\end{array}$ & $3.6(1.5)$ & $7.0(2.0)$ & $3.4(2.8$ to 3.9) \\
\hline
\end{tabular}

the intravenous group and a $75 \%$ cure rate in the switch group $(\alpha=0.05$, two sided; $1-\beta=0.80)$. We calculated the absolute difference in cure rate including $95 \%$ confidence interval. Equivalence was rejected if the lower limit of the confidence interval exceeded $-10 \%$. We performed intention to treat analysis and per protocol analysis.

\section{Results}

\section{Characteristics of the patients and treatment} assignment

Patients were enrolled between July 2000 and March 2004. Enrolment was slow and only 302 patients were randomised: 150 to the control group to receive a standard course of seven days' intravenous treatment and 152 to the early switch group. Baseline characteristics were similar in both groups. More than $80 \%$ of patients were in pneumonia severity class IV or V. Most patients received empirical monotherapy with amoxicillin or amoxicillin plus clavulanic acid $(\mathrm{n}=174 ; 58 \%)$ or a cephalosporin $(\mathrm{n}=59 ; 20 \%)$. The most frequently identified micro-organism was Streptococcus pneumoniae $(\mathrm{n}=76 ; 25 \%)$. Atypical pathogens were detected in 33 patients $(11 \%)$. Before day 3, $37(12 \%)$ patients were excluded from analysis, leaving 132 patients for analysis in the intervention group and 133 in the control group. After three days of intravenous treatment, 108/ $132(81 \%)$ patients in the intervention group were switched to oral treatment, of whom $102(94 \%)$ received amoxicillin plus clavulanic acid. See bmj.com for details.

In the control group, five patients did not receive intravenous antibiotics for all seven days because of phlebitis associated with intravenous treatment. Overall duration of antibiotic treatment was 10.1 days in the intervention group and 9.3 days in the control group (mean difference -0.8 days, $95 \%$ confidence interval -0.6 to 2.0

\section{Clinical outcome}

In intention to treat analysis, treatment failed in 22 $(17 \%)$ and $20(15 \%)$ patients in the intervention group and the control group $(-2 \%,-10 \%$ to $7 \%$; table 1$)$. In the control group, nine (7\%) patients were still in hospital on day 28 , eight $(6 \%)$ had deteriorated clinically, and eight $(6 \%)$ had died. In the intervention group, six $(5 \%)$ patients were still in hospital, six $(5 \%)$ had deteriorated clinically, and five (4\%) had died.

The duration of intravenous treatment was significantly shorter in the intervention group (table 1). Average time to meet the discharge criteria was 5.2 (2.9) days in the intervention group and 5.7 (3.1) days in the control group ( 0.5 days, -0.3 to 1.2 ). Total length of hospital stay was 9.6 (5.0) and 11.5 (4.9) days for patients in the intervention group and control group (1.9 days, 0.6 to 3.2). Per protocol analysis showed comparable results for clinical outcome and length of hospital stay (see bmj.com). Patients meeting discharge criteria were not always discharged immediately; the main reasons were incomplete resolution of all clinical criteria of pneumonia or comorbid illness, the anticipated lack of continued care after discharge, and doctor's considerations.

Overall, patients treated with oral or intravenous antibiotics had the same problems with regard to mobility and other side effects. 


\section{Discussion}

Patients admitted to hospital with severe community acquired pneumonia can be managed more efficiently by an early switch from intravenous to oral drugs- $-81 \%$ of patients could be switched to oral antibiotics on day 3 , which reduced the average length of hospital stay by at least 1.9 days. Our findings can probably be generalised, as our patients were similar to other cohorts with this disease in terms of average length of stay in the control group, aetiology, and mortality rate.

\section{Strengths and limitations}

In most previous studies, a non-randomised design was used, specific patient populations in military hospitals or moderately ill patients were studied, sample sizes were small, or the patients were switched relatively late.

Our study had several limitations. The number of patients included was lower than calculated before the start of the study. Because results show small and nonsignificant differences in rates of treatment failure, however, it is highly unlikely that an early switch is more than $10 \%$ less effective. Moreover, mortality rates were even lower in the intervention group.

The effects of switching treatment may have been overestimated for two reasons. Firstly, in patients who were clinically stable at day 3 , protocol dictated an intravenous to oral switch, but it is uncertain how many patients would have been switched in daily practice. With growing confidence in the safety of an early switch strategy this effect might decrease. Secondly, the minimum duration of intravenous treatment for the control group of seven days was also dictated by protocol, and shorter durations would have decreased the benefits of an early switch as long as failure rates remained the same.

In contrast, the effects of switching treatment could have been underestimated for two reasons. Firstly, the protocol did not cover discharge, and doctors' views on continued stay in hospital strongly influenced delayed discharge of clinically stable patients. With growing confidence, this phenomenon may decrease. Although clinical instability at discharge is associated with adverse clinical outcomes, clinical deterioration after reaching clinical stability is rare. ${ }^{6}$ In our study, only three $(2 \%)$ patients were restarted on intravenous drugs after being switched to oral treatment. Secondly, the protocol only allowed patients to be switched to oral drugs when they were clinically stable on day 3 .

\section{What is already known on this topic}

An early switch to treatment with oral antibiotics in community acquired pneumonia may allow early discharge and reduce drug and treatment costs

Studies have evaluated only patients with mild to moderately severe disease, rarely in a randomised design

\section{What this study adds}

Early transition to oral antibiotics can safely be implemented in clinical practice in patients with severe community acquired pneumonia who do not need treatment in intensive care

Such a strategy leads to a reduced length of hospital stay

Patients clinically stable before day 3 could possibly be switched earlier, which could enhance the benefits of this strategy.

Funding: Dutch Health Insurance Council, grant OG 99-64.

Contributors: See bmj.com.

Competing interests: None declared.

Ethical approval: Ethics committees of participating institutions. The study was presented in part at the Interscience Conference on Antimicrobial Agents and Chemotherapy, abstract O-1607, Washington DC, USA, 2004.

1 Weingarten SR, Riedinger MS, Hobson P, Noah MS, Johnson B. Evaluation of a pneumonia practice guideline in an interventional trial. Am J Respir Crit Care Med 1996;153:1110-5.

2 Van Kasteren MEE, Wijnands WJ, Stobbering EE, Janknegt R, van der Meer JW. Optimization of the antibiotics policy in the Netherlands. II. Meer JW. Optimization of the antibiotics policy in the Netherlands. II. SWAB guidelines for the antimicrobial therapy of pneumonia in patients at home and as nosocomial infections. The Netherlands Antibiotic Policy

Foun AW, Hedl CB, Klein

3 Chow AW, Hall CB, Klein JO, Kammer RB, Meyer RD, Remington JS. General guidelines for the evaluation of new anti-infective drugs for the treatment of respiratory tract infections. Clin Infect Dis 1992;15(suppl 1):s62-88.

4 American Thoracic Society. Guidelines for the management of adults with community acquired pneumonia. Am J Respir Crit Care Med 2001;163:1730-54.

5 Fine MJ, Auble TE, Yealy DM, Hanusa BH, Weissfeld LA, Singer DE, et al. A prediction rule to identify low-risk patients with community acquired pneumonia. N Engl J Med 1997;336:243-50.

6 Halm EA, Fine MJ, Kapoor WN, Singer DE, Marrie TJ, Siu AL. Instability Halm EA, Fine MJ, Kapoor WN, Singer DE, Marrie TJ, Siu AL. Instability
on hospital discharge and the risk of adverse outcomes in patients with pneumonia. Arch Intern Med 2002;162:1278-84.

(Accepted 22 September 2006)

doi 10.1136/bmj.38993.560984.BE

\section{Junior doctor identity crises}

I have noticed a curious trend occurring in my hospital over the past six months: junior doctor identity crisis. As medical students we were constantly reminded to inform patients of our status, and we did this readily. We were proud to wear badges inscribed "medical student" to avoid any misunderstandings. Towards the end of our undergraduate education we could not wait until we would be able to introduce ourselves as "doctor."

However, it has turned out that confusion starts with graduation, the new training structure for junior doctors in the UK producing various new titles. The identity badges of junior medical staff are now littered with ambiguous job titles such as
"F1 doctor," "F1 PRHO," "SHO," "F2 Doctor," "FY2 doctor," and so on. How will our patients respond to this?

Will an "FY2 SHO doctor" be assumed more senior than an "ST2 doctor"? How will patients respond to the title of "specialist trainee"? Most are currently aware that registrars are more senior than senior house officers, and in a few years it will be probably be the same with the new titles. I propose all name badges have the doctor's name followed by, for example, "doctor-ST3." Until then, it is our responsibility to take extra care in explaining our status, just as we did as students.

Andy Potter senior house officer, Milton Keynes General Hospital (apotter1980@hotmail.com) 